\title{
Epidemiology, Symptoms, and Treatment Characteristics of Hyponatremic Psychiatric Inpatients
}

\author{
Christian Lange-Asschenfeldt, MD, PhD, * Georg Kojda, PharmD, PhD, $\dagger$ \\ Joachim Cordes, MD, * Florence Hellen, MD, * Andreas Gillmann, MD, * \\ Renate Grohmann, MD, $\neq$ and Tillmann Supprian, $M D, P h D^{*}$
}

\begin{abstract}
Hyponatremia is a common phenomenon in psychiatry occurring as an adverse effect to drugs or following polydipsia. We performed a retrospective in-depth analysis of hyponatremia cases in a large unselected population of psychiatric inpatients. During a 3-year period, all cases of hyponatremia were identified among patients admitted to a large psychiatric state and university hospital by the institution's electronic laboratory database. Demographic, treatmentrelated, and laboratory data were obtained by consecutive chart review, respectively. Hyponatremia occurred in 347 (4.9\%) of 7113 cases, of which the majority (78\%) displayed only a mild manifestation. Symptoms were recorded in $28.8 \%$ of cases, already occurred in mild forms, and comprised gait impairment (45\%, including falls), confusion (30\%), sedation (26\%), and dyspepsia (41\%). Age, female sex, nonpsychiatric drug polypharmacy - particularly with thiazides and/or angiotensinconverting enzyme inhibitors - and diagnosis of a mood disorder were associated with more severe hyponatremia, respectively. The proportion of hyponatremic patients treated with venlafaxine, trazodone, carbamazepine, oxcarbazepine, and first-generation antipsychotics, respectively, was significantly higher in the hyponatremia sample than in the normonatremic population. This was, surprisingly, not the case with selective serotonin reuptake inhibitors or any other antidepressant drug class. We found prescription with second-generation antipsychotics to be significantly associated with less severe hyponatremia.

Hyponatremia may be mainly attributed to the syndrome of inappropriate antidiuretic hormone secretion, as indicated by decreased serum osmolarity in our sample. Besides old age and female sex, treatment with certain drugs - rather than whole drug classes - carries a substantially increased risk.
\end{abstract}

Key Words: hyponatremia, SIADH, prevalence, psychiatric inpatients, drug treatment

(J Clin Psychopharmacol 2013;33: 799-805)

$\mathrm{H}^{\mathrm{s}}$ yponatremia, usually defined as a serum sodium concentration $\left(\left[\mathrm{Na}^{+}\right]\right)$below $135 \mathrm{mEq} / \mathrm{L}$, is the most prevalent electrolyte aberrance encountered in hospitalized patients in both general medical and psychiatric institutions. ${ }^{1,2}$ Signs and

From the Departments of *Psychiatry and Psychotherapy and $\dagger$ Pharmacology and Toxicology, Medical Faculty, Heinrich Heine University, Düsseldorf; and \$Department of Psychiatry and Psychotherapy, Ludwig Maximilians University, Munich, Germany.

Received June 1, 2012; accepted after revision March 1, 2013.

Reprints: Christian Lange-Asschenfeldt, MD, PhD, Klinik und Poliklinik für Psychiatrie und Psychotherapie, Heinrich-Heine-Universität-LVRKlinikum Düsseldorf, Bergische Landstr. 2, D-40629 Düsseldorf, Germany (e-mail: kn50050@lvr.de).

Supplemental digital content is available for this article. Direct URL citation appears in the printed text and is provided in the HTML and PDF versions of this article on the journal's Web site (www.psychopharmacology.com). Copyright (C) 2013 by Lippincott Williams \& Wilkins

ISSN: 0271-0749

DOI: 10.1097/JCP.0b013e3182a4736f symptoms mainly entail neuropsychiatric and gastrointestinal manifestations. Hyponatremia has been linked with increased health care resource use and costs. ${ }^{3}$ It can cause significant morbidity and, by way of an osmotic demyelinating encephalopathy, may lead to serious consequences, such as lethargy, confusion, coma, and occasionally death. ${ }^{4,5}$ However, clinical symptoms vary and depend mainly on severity and abruptness of onset. Overt symptoms generally do not appear until $\left[\mathrm{Na}^{+}\right]$falls below $130 \mathrm{mEq} / \mathrm{L} .{ }^{6}$ Among psychiatric patients, hyponatremia is believed to be frequently underdiagnosed because clinical signs may mimic psychopathological symptoms of the underlying condition. ${ }^{7}$

Physiological serum $\left[\mathrm{Na}^{+}\right]$is maintained within tight limits through a complex homeostatic system involving hypothalamic osmoreceptors and peripheral baroreceptors regulating water intake and urine output via changes in thirst and the neurohypophyseal secretion of the antidiuretic hormone arginine-vasopressin (AVP). ${ }^{8}$ Thus, excessive water intake or inappropriate release of AVP in the absence of osmotic stimuli, respectively are among the most common pathomechanisms of hyponatremia. The latter condition is known as the syndrome of inappropriate antidiuretic hormone secretion (SIADH) and occurs, among an array of other causes, as an adverse effect of a large number of psychotropic and other drugs. ${ }^{7,9,10}$ Above all, a wealth of case reports, casecontrol, and population-based studies have implicated antidepressants, such as tricyclic antidepressants (TCAs), selective serotonin reuptake inhibitors (SSRIs), selective serotonin and norepinephrine reuptake inhibitors (SNRIs), and certain anticonvulsants - mainly carbamazepine and oxcarbazepine - in hyponatremia of this origin. ${ }^{1-13}$ The most prevalent nonpsychiatric drugs associated with SIADH-induced hyponatremia are thiazide diuretics and angiotensin-converting enzyme inhibitors (ACEIs), among others. ${ }^{7}$

Age and female sex are established major risk factors for treatment-emergent hyponatremia. Moreover, in psychiatry, special patient populations are at particular risk to develop this type of electrolyte disturbance. These include mainly schizophrenia patients with polydipsia or others with compulsive drinking behavior and individuals with anorexia-related surreptitious water loading. ${ }^{14}$ Occurrence of polydipsia and polyuria in schizophrenia were reported already in the early part of the 20th century, before specific psychiatric drug treatment became available. ${ }^{15}$ The prevalence of hyponatremia has been estimated as $15 \%$ and $11 \%$ in general and psychiatric hospital settings, respectively. ${ }^{16,17}$ However, numbers vary considerably, depending on threshold definitions and subpopulations under analysis. Despite the apparent clinical significance of the issue, our knowledge on prevalence, risk factors, and drugs involved in treatment-related hyponatremia is mainly confined to antidepressants, in particular SSRIs and venlafaxine. ${ }^{18,19}$ Moreover, it still relies to a large extent on anecdotal data from case studies and the investigation of small patient cohorts in special subpopulations. $^{2}$ 
The objective of the present study was thus to analyze in an unselected population of hospitalized psychiatric patients the frequency and risk factors as well as the clinically most relevant symptoms of hyponatremia. We carried out an in-depth analysis of a complete set of hyponatremia cases that occurred during inpatient treatments of 3 consecutive years in a large psychiatric state and university hospital.

\section{MATERIALS AND METHODS}

\section{Study Design}

Data for this retrospective analysis were drawn from records of all patients of the Department of Psychiatry and Psychotherapy, University Hospital, Düsseldorf, Germany, who were hospitalized from January 1, 2004, through December 31, 2006. Quality standards in this institution require close monitoring of routine laboratory parameters, including serum $\left[\mathrm{Na}^{+}\right]$, in all patients receiving psychiatric drug treatment. All cases of hyponatremia (serum $\left[\mathrm{Na}^{+}\right]<135 \mathrm{mEq} / \mathrm{L}$ ) that occurred during this time episode were identified using the hospital's electronic laboratory database. The first pathological serum $\left[\mathrm{Na}^{+}\right]$measured during the treatment course in a given case was referred to as "index hyponatremia." A thorough chart analysis of all hyponatremia cases was undertaken. Basic demographic variables as well as the following relevant illness- and treatmentrelated data were obtained:

- complete psychiatric and medical diagnoses;

- complete psychiatric and medical drugs prescribed at time point of index hyponatremia;

- serum $\left[\mathrm{Na}^{+}\right](\mathrm{mEq} / \mathrm{L})$ at time point of index hyponatremia and subsequent values in case of serial measurements;

- other laboratory parameters: serum levels of potassium $(\mathrm{mEq} / \mathrm{L})$, urea $(\mathrm{mg} / \mathrm{dL})$, glucose $(\mathrm{mg} / \mathrm{dL})$, and estimated glomerular filtration rate (eGFR) at time point of index hyponatremia; and

- serum osmolarity, calculated according to Bhagat et $\mathrm{al}^{20}: 1.86 \times$ $\left[\mathrm{Na}^{+}\right](\mathrm{mEq} / \mathrm{L})+0.056 \times$ serum glucose $(\mathrm{mg} / \mathrm{dL})+0.17 \times$ serum urea $(\mathrm{mg} / \mathrm{dL})+9$.

For assessment of symptoms, information was extracted from the daily follow-up reports of nursing staff and treating physicians from 2 days before until 5 days after the date of index hyponatremia. Symptoms were operationalized into the following entities: (i) dizziness/clouding of consciousness, (ii) psychomotor retardation, (iii) confusion, (iv) gait disturbance, (v) fall events, (vi) seizure activity, and (vii) gastrointestinal symptoms (nausea/vomiting, diarrhea). Symptoms were considered only if, according to the chart documentation, they had occurred in close temporal relation to the index hyponatremia and if they were distinct from those of the psychopathology of the underlying psychiatric condition of the patient.

To determine the hyponatremia prevalence of single compounds and drug classes, the number of prescriptions of the respective substance in the hyponatremia sample had to be related to that in the inpatient population of the defined 3-year investigation period. Because exact information on prescription rates of the whole population was impossible to obtain, prescription rates were estimated using the method of the psychiatric drug surveillance program AMSP ("Arzneimittelsicherheit in der Psychiatrie") in which the hospital participates. ${ }^{21}$ In this project, the data on drug use are derived from 2 reference days per year (ie, 6 reference days within the 3 -year period). On these reference days, all administered drugs and their dosages are recorded for all patients of the institution. From these data, estimated prescription rates for the whole population are calculated for compounds and drug classes of interest. ${ }^{22}$ We focused our analysis on antidepressants, high-potency first-generation antipsychotics (FGAs), second-generation antipsychotics (SGAs), and mood stabilizers.

\section{Statistical Analysis}

The hyponatremia prevalence of a given drug/drug class was determined as the number of cases found to be treated with this drug/drug class in the hyponatremia sample N[hypo], divided by the number of patients exposed to this drug/drug class in the whole population under study (N[total]). For comparison of hyponatremia prevalences between different drugs/drug classes, prevalences were compared on a one-by-one basis with the average hyponatremia prevalence of all other drugs, respectively, using the $\chi^{2}$ test or Fisher exact test, as appropriate. Data are depicted together with their $95 \%$ confidence intervals (95\% CIs)

Other data were provided as mean $\pm \mathrm{SD}$ or median/range, as appropriate, for continuous variables or in terms of number and percentage for categorical variables, respectively. For analysis of the relation of $\left[\mathrm{Na}^{+}\right]$to various demographic, treatmentrelated and laboratory parameters, $\left[\mathrm{Na}^{+}\right]$values of the hyponatremia case sample were collapsed into quintiles, with cases displaying the lowest $\left[\mathrm{Na}^{+}\right]$appearing in the fifth quintile. Continuous variables of interest were compared across $\left[\mathrm{Na}^{+}\right]$ quintiles using analysis of variance or the Kruskal-Wallis rank sum test, as appropriate. Dunn post hoc test was used in case of multiple comparisons. Categorical variables were analyzed with the $x^{2}$ test with Yates correction. Correlations were calculated using the Spearman rank correlation coefficient. Significance was set at $P<0.05$.

\section{RESULTS}

\section{Demographic and IIIness-Related Data}

From 2004 through 2006, a total of N[total] = 7113 psychiatric inpatient treatments were registered in our hospital. Hyponatremia $\left(\left[\mathrm{Na}^{+}\right]<135 \mathrm{mEq} / \mathrm{L}\right)$ was found in $\mathrm{N}[\mathrm{hypo}]=347$ cases $(4.9 \%)$. The median age of the case sample was 67 years (range, 21-101 years). There was a predominance of elderly subjects ( $\geq 60$ years) in $\mathrm{N}$ [hypo] (odds ratio, $11.11 ; 95 \% \mathrm{CI}$, $8.82-14.0 ; P<0.0001)$. Among the subgroup of patients 60 years or older in $\mathrm{N}$ [total], the hyponatremia prevalence was $16 \%$. Whereas the sex proportion of nonhyponatremic cases was rather balanced, women prevailed in N[hypo] (odds ratio, 1.78; 95\% CI, 1.42-2.23; $P<0.0001$ ).

Most prevalent psychiatric diagnoses in hyponatremia cases were mood disorders $(60 \%, \mathrm{n}=209$; mainly depression $)$, followed by substance-related disorders $(\mathrm{n}=118,34 \%)$ with a proportion of $89 \%$ with dual diagnoses $(n=105)$, schizophrenia and schizoaffective disorders $(32 \%, \mathrm{n}=112)$, dementias and other organic psychiatric disorders $(n=107,31 \%)$, and others $(n=21,6 \%)$. Twenty-three percent of cases $(n=178)$ had more than 1 psychiatric diagnoses. More than three-fourths (78\%) of cases had mild ([Na $]$ : $130-134 \mathrm{mEq} / \mathrm{L})$ and $20 \%$ had moderate ( $\left[\mathrm{Na}^{+}\right]$: $120-129 \mathrm{mEq} / \mathrm{L}$ ) hyponatremia. Only a small minority of cases $(2 \%)$ displayed severe hyponatremia $\left(\left[\mathrm{Na}^{+}\right]<120 \mathrm{mEq} / \mathrm{L}\right)$. The mean $\left[\mathrm{Na}^{+}\right]$of the $\mathrm{N}[$ hypo] was $131.2 \pm 3.9 \mathrm{mEq} / \mathrm{L}$.

Symptoms were recorded in 100 cases $(28.8 \%)$ (Table 1). The mean $\left[\mathrm{Na}^{+}\right]$of symptomatic patients was $131.0 \pm 4.0 \mathrm{mmol} / \mathrm{L}$ and almost identical to that of the complete sample. There was no difference regarding age and sex distribution among symptomatic versus asymptomatic hyponatremia cases. As expected, symptom occurrence depended on the severity of hyponatremia (Supplementary Table A, Supplemental Digital Content 1, http://links.lww.com/JCP/A209). Neuropsychiatric symptoms 
TABLE 1. Symptoms of Hyponatremia Cases

\begin{tabular}{|c|c|c|c|}
\hline Symptom & $\mathbf{n}$ & $\% *$ of Patients With Symptoms $(n=100)$ & $\%$ of Patients With Hyponatremia $(n=347)$ \\
\hline \multicolumn{4}{|l|}{ Neuropsychiatric } \\
\hline Dizziness/clouding of consciousness & 26 & 26 & 7.5 \\
\hline Psychomotor retardation & 14 & 14 & 4.0 \\
\hline Confusion & 30 & 30 & 8.6 \\
\hline Gait impairment & 45 & 45 & 12.9 \\
\hline Falls & 15 & 15 & 4.4 \\
\hline Seizures & 4 & 4 & 1.2 \\
\hline \multicolumn{4}{|l|}{ Gastrointestinal } \\
\hline Nausea/vomiting/diarrhea & 41 & 41 & 11.8 \\
\hline
\end{tabular}

such as gait impairment, confusion, dizziness/clouding of consciousness, and psychomotor slowing were recorded. Gait impairment, including falls, was the most prevalent symptom. The respective chart documentations included predominantly trouble maintaining balance or climbing stairs and reduced gait speed and stride length, among others. Seizures occurred in only $1.2 \%$ of cases. Gastrointestinal symptoms (dyspepsia) were also noted in a considerable proportion of cases.

\section{Serum $\left[\mathrm{Na}^{+}\right]$and Characteristic Baseline, Treatment-Related, and Laboratory Parameters}

The median (interquintile range) or mean \pm SD values of salient demographic, treatment-related, and chemical laboratory variables of the case sample by quintiles of $\left[\mathrm{Na}^{+}\right]$are provided in Supplementary Table A (Supplemental Digital Content 1). Patients in the fifth quintile were significantly older and more often women. Interestingly, low $\left[\mathrm{Na}^{+}\right]$was associated with a shorter duration of treatment, probably because of early referral of symptomatic hyponatremic patients to other (medical) departments. Regarding major Axis I psychiatric diagnoses, there was no association between hyponatremia and a diagnosis of schizophrenia/schizoaffective disorder or a substance use disorder, respectively. However, patients with low $\left[\mathrm{Na}^{+}\right]$were significantly more often diagnosed with a mood disorder (Supplementary Table A, Supplemental Digital Content 1).

The mean serum osmolarity in the sample was $265.3 \mathrm{mOsm} / \mathrm{kg}$ $\mathrm{H}_{2} \mathrm{O}$, which is below the physiological lower limit of $275 \mathrm{mOsm} / \mathrm{kg}$ $\mathrm{H}_{2} \mathrm{O}$. ${ }^{9}$ Serum osmolarity was negatively correlated with the eGFR $(r=-0.42 . P<0.0001)$. These results may point toward SIADH as the main overall underlying cause of hyponatremia in the case sample. In accordance, low serum osmolarity was significantly associated with the degree of severity of hyponatremia as evidenced by Supplementary Table A (Supplemental Digital Content 1). Of the various chemical parameters under study, only serum chloride concentration $\left[\mathrm{Cl}^{-}\right]$correlated with $\left[\mathrm{Na}^{+}\right]$. There was no significant association with serum potassium concentration $\left[\mathrm{K}^{+}\right]$, eGFR, urea, glucose, or hematocrit across $\left[\mathrm{Na}^{+}\right]$quintiles, respectively (Supplementary Table A, Supplemental Digital Content 1).

\section{Hyponatremia Prevalences of Psychiatric and Medical Drugs and Drug Classes}

There was no difference regarding the prescribed number of total and psychiatric drugs across quintiles. However, the number of nonpsychiatric drugs (mainly ACEI, thiazide diuretics, opiate analgesics, nonsteroidal anti-inflammatory drugs, proton pump inhibitors) was significantly higher in the fifth $\left[\mathrm{Na}^{+}\right]$quintile (Supplementary Table A, Supplemental Digital Content 1). Of interest, regarding the proportions of relevant psychiatric drugs and drug classes found across $\left[\mathrm{Na}^{+}\right]$quintiles, no significant difference was observed apart from a lower prescription rate of SGAs in the lowest $\left[\mathrm{Na}^{+}\right]$quintile. By contrast, a significant impact of nonpsychiatric drugs on hyponatremia severity was found. Patients at the lower end of $\left[\mathrm{Na}^{+}\right]$were significantly more often treated with ACEIs and combinations of ACEIs and thiazide diuretics, respectively, and also showed a strong trend of a higher treatment frequency with thiazides alone. Taken together, prescriptions with medical drugs, especially ACEIs and thiazide diuretics, and medical combination therapy seem to convey a particular risk to develop more severe degrees of hyponatremia. This was not the case for any psychotropic drug or drug class, including SSRIs. Moreover, polypharmacy with thiazides and/or ACEI was found significantly more often in SSRI-treated cases in the fifth (lowest $\left[\mathrm{Na}^{+}\right]$) versus first quintile $(P<0.0023$; data not depicted).

To estimate overall hyponatremia prevalences for salient psychotropic substances, we related the proportion of a given compound/drug class found in the hyponatremia sample N[hypo] to that calculated for the nonhyponatremic part of the whole population N[total], respectively. The results are shown in Figure 1. Because of small case numbers for the majority of single drugs, most data were merged to major drug groups and are presented as a whole.

\section{Antidepressants}

Treatment with the TCAs amitriptyline, clomipramine, doxepine, nortriptyline, trimipramine, and the tetracyclic antidepressant maprotiline was documented in the hyponatremia sample N[hypo]. As depicted in Figure 1A, TCAs showed a hyponatremia prevalence of overall $4.5 \%$, which was not significantly different from the average of all other drugs. Of note, regarding the SSRIs (3.5\%), which have been implicated in hyponatremia in many case reports and some retrospective and cohort studies, the hyponatremia prevalence did not exceed the average prevalence of all other psychotropic drugs. In contrast, venlafaxine treatment was significantly more prevalent among the hyponatremic sample (Fig. 1A). Of the remaining antidepressants not associated with one of the mentioned classes, mirtazapine had an average and trazodone had a surprisingly high hyponatremia rate. However, the $95 \%$ CI for the latter was wide, and the number of treated patients was relatively small, so this finding should be interpreted with caution. 
A

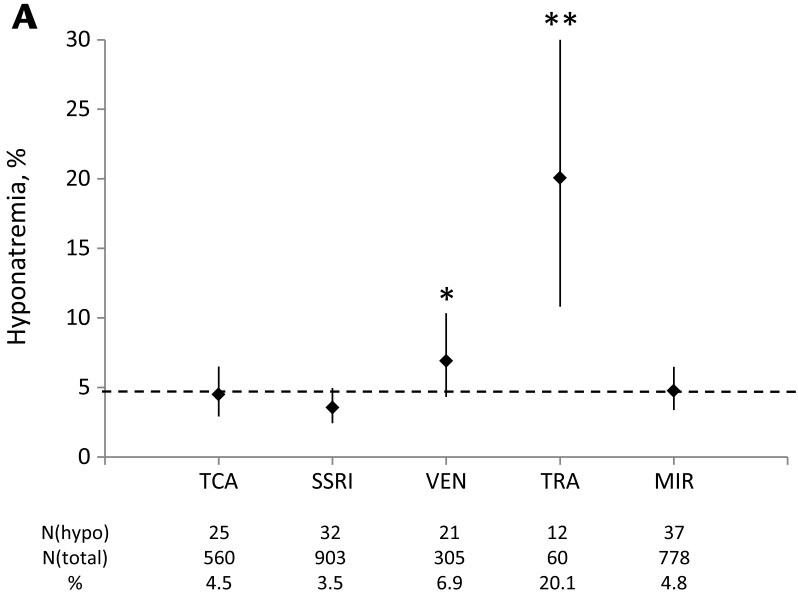

B

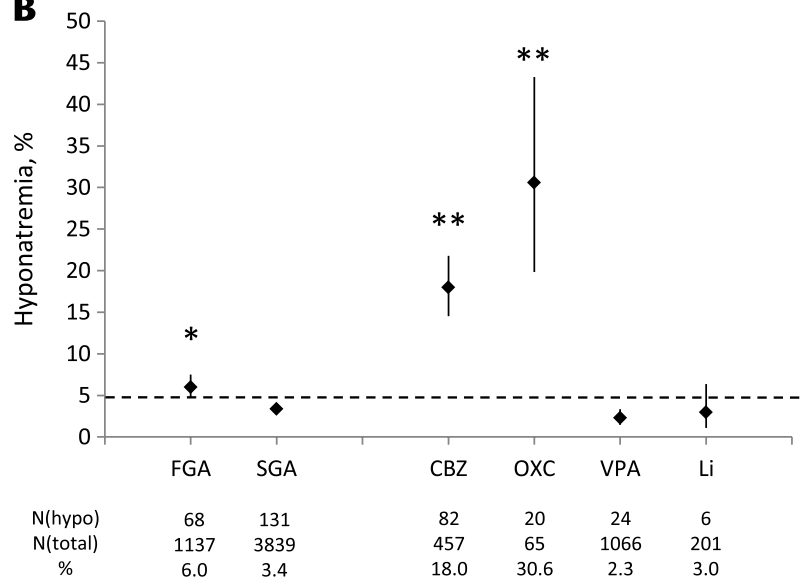

FIGURE 1. Prevalences of hyponatremia associated with various psychotropic drugs/drug classes in psychiatric inpatients. A, Antidepressants and (B) antipsychotics and mood stabilizers. The hyponatremia prevalence of a given drug/drug class was determined as its number in the hyponatremia sample (N[hypo]) related to the number of patients exposed to the respective drug/drug class in the whole study population (N[total]). Values are shown together with their $95 \%$ Cls. The hyponatremia prevalence of a given drug/drug class was compared with that of all others, respectively ( $\chi^{2}$ or Fisher exact test, as appropriate). Thus, the comparator's mean of a respective drug may differ from the group mean of all drugs (indicated by the dotted line for orientation), which explains why, in case of venlafaxine, the group mean lies within the depicted $95 \% \mathrm{Cl}$. $* P<0.05$ $* * P<0.01$. CBZ indicates carbamazepine; Li, lithium; MIR, mirtazapine; OXC, oxcarbazepine; TRA, trazodone; VEN, venlafaxine; VPA, valproate.

\section{Antipsychotics}

"Conventional" high-potency FGAs were associated with a significantly higher hyponatremia rate in the case sample compared with an average rate for SGAs (Fig. 1B). Moreover, SGA prescription was associated with less severe hyponatremia.

\section{Mood Stabilizers}

According to expectations, oxcarbazepine and carbamazepine had the highest hyponatremia rates in this group and one of the highest overall. Hyponatremia was significantly less common with valproate and almost average with lithium (Fig. 1B).

\section{DISCUSSION}

\section{Methodological Issues}

We have investigated a complete data set derived from a large and unselected psychiatric inpatient population, which we consider a strength of this report. Furthermore, salient epidemiological and treatment-related data and particularly elaborate chart documentation on symptoms were made available to draw meaningful conclusions regarding various clinically relevant aspects of the topic. However, as the study is retrospective, it has inherent shortcomings. Most relevant, the frequent use of multiple medications and the presence of concomitant medical illnesses make causal relationships difficult to establish. Hyponatremia in psychiatry is a complex and multifaceted phenomenon that may occur as an adverse drug reaction as well as in close relation to certain diagnoses, above all as a result of polydipsia in schizophrenia, a frequently neglected and certainly underdiagnosed phenomenon. ${ }^{2}$ High normalized diurnal weight gain, concomitant low urinary osmolality, and of course increased daily fluid intake are indicative of polydipsia. Because these features were not regularly available in the charts, we focused our analysis on other treatment-related parameters, mainly drug exposure, being aware that lack of consideration of polydipsia as a potential confounding factor is a limitation to our study. We thus tried to take care not to draw direct causal linkages to drug treatment but rather provide a descriptive approach.

Because of the large population size, we were not able to list the drugs prescribed to individual patients in the complete population N[total]. Thus, we had to estimate prescription rates of the drugs in question (as detailed in Materials and Methods), which may be viewed as another limitation. This method, however, as established within the framework of the AMSP program, has been used successfully in previous retrospective drug safety studies. ${ }^{21,23}$

Regarding symptom analysis, we had to rely on staff documentation at the time point of the first pathological $\left[\mathrm{Na}^{+}\right]$ defined as index hyponatremia. This may have led to underestimation of symptom severity, which may have increased parallel to progression of hyponatremia in the further course.

\section{General and Epidemiological Considerations}

In this study, we provide a retrospective analysis of a large unselected population of psychiatric inpatients, spanning a 3-year period in a primary and secondary psychiatric care environment and using $135 \mathrm{mEq} / \mathrm{L}$ as the low normal limit of $\left[\mathrm{Na}^{+}\right]$. The hyponatremia prevalence was $4.9 \%$ in the overall population and $16 \%$ in the elderly subpopulation, with mild manifestations in the majority of cases. In the literature, estimates of hyponatremia prevalence depend on clinical circumstances (eg, medical, surgical, psychiatric, elderly patients, etc) and on predefined cutoff levels. Thus, data in general hospital settings widely range from $2.5 \%$ to $30 \%{ }^{24}$ A recent systematic review revealed a prevalence of $6.0 \%$ in normal and $22.2 \%$ in geriatric wards, respectively. ${ }^{25}$ In psychiatry, data exist mainly on specialized psychiatric populations, such as schizophrenia patients with polydipsia $(25 \%),{ }^{17}$ older patients treated with SSRIs or venlafaxine $(39 \%),{ }^{18}$ and inpatients of psychogeriatric wards $(7.7 \%)$

In contrast to the whole inpatient population, the majority of cases in the hyponatremia sample was 60 years or older, and age correlated with hyponatremia severity. This is consistent with the current literature where age is an established risk factor for hyponatremia, ${ }^{1,2,9,23}$ probably due to multiple reasons. For example, older patients are more likely to have multiple medical 
diagnoses and thus are prescribed more drugs, including such with known potential to induce hyponatremia such as ACEI, thiazides, and so on. Moreover, age-related alterations of renal function impede water homeostasis and sodium metabolism causing reduced drug clearance. ${ }^{26}$ The vast majority of hyponatremia cases in the elderly ${ }^{27}$ and most of the cases occurring in psychiatric patients ${ }^{2}$ have been attributed to SIADH. In the present study, we found an association of hyponatremia severity and low serum osmolarity indicative of the presence of SIADH.

We found the likelihood of hyponatremia approximately doubled for female patients. It is known that hyponatremia in general and drug-induced hyponatremia in particular disproportionally affect women. ${ }^{7}$ The exact underlying cause of this finding is still unknown. However, a variety of sex differences of the physiological sodium and water metabolism exists, which may depend at least in part on ovarian hormone activity. It is known, for example, that the antidiuretic responsiveness to AVP and its analog on desmopressin is higher in women than in men. ${ }^{28}$ In addition, sex-related variances in the function of several sodium transport systems across membranes have been found, resulting in differences in intracellular and extracellular $\left[\mathrm{Na}^{+}\right]$, favoring a relative state of lower extracellular $\left[\mathrm{Na}^{+}\right]$and a higher sensitivity to drug-induced hyponatremia in women. ${ }^{29}$

\section{Symptoms}

Confusion, sedation, gait impairment, and (rarely) seizures were neuropsychiatric symptoms recorded in our sample, indicating the presence of osmotic encephalopathy at least in a considerable proportion of cases. ${ }^{9}$ In addition, gastrointestinal symptoms were common. Gait impairment (including falls) was the most prevalent condition in our sample. This finding seems to be of particular clinical importance because subtle gait impairment may easily be overlooked, and special precautions may prevent significant consecutive morbidity and socioeconomic consequences. ${ }^{30,31}$ In fact, it has been shown earlier that even chronic hyponatremia conveys a considerable risk of falls due to gait and attention impairment. ${ }^{32}$ As expected, symptom occurrence recorded in the charts clearly correlated with hyponatremia severity. Nevertheless, we found a considerable proportion of symptomatic cases already in mild forms confirming previous observations. ${ }^{33,34}$ We are aware that cases with $\left[\mathrm{Na}^{+}\right]$of greater than $130 \mathrm{mEq} / \mathrm{L}$ may in part represent a variation of normality. However, we have included them in our analysis because of overwhelming evidence of an increased independent risk of adverse outcome and even mortality with mild and very mild hyponatremia. ${ }^{35-37}$

\section{Association of Hyponatremia With Psychotropic Compounds and Drug Classes}

One of the aims of our study was to relate the presence of hyponatremia in a complete case sample during a defined period to the treatment with psychotropic drugs of interest. As already pointed out, our retrospective data were not suitable to causally link hyponatremia with certain drug treatments in our case sample because of frequent polypharmacy and the impossibility to exclude underlying etiological factors that are diagnosis related. Our approach was thus mainly descriptive; however, it may at least allow for indirect conclusions. Indeed, despite this limitation and as outlined below, salient results are well suited to findings in the current literature regarding the risk of hyponatremia pertaining to particular drugs or drug groups.

Drug-induced hyponatremia may occur (1) secondary to SIADH by inducing excess hypothalamic production and hypophyseal release of AVP without the appropriate physiological stimuli (ie, antidepressants, antipsychotic drugs), ${ }^{2}(2)$ through potentiation of the endogenous AVP effect at the renal tubule level (ie, several anticonvulsants/mood stabilizers), (3) in response to reduction of the threshold for AVP secretion by resetting the osmostat, and (4) increased urinary loss of sodium (ie, diuretics, mainly thiazides). ${ }^{7}$ The hyponatremia risk seems to be higher when multiple mechanisms are involved. Carbamazepine and oxcarbazepine, for example, can elicit hyponatremia via mechanisms (1), (2), and (3) ${ }^{38-42}$ Accordingly, in the present study, the hyponatremia prevalence of these compounds was 3- and 6-fold increased compared with the average, respectively. Values were almost identical to those found in a previous study in epilepsy patients $(13.5 \%$ and $29.9 \%$ in our work, respectively). ${ }^{43}$ Thus, hyponatremia seems to be a common adverse effect of these anticonvulsants, independent of diagnosis. In contrast, valproate and lithium displayed much lower hyponatremia rates in our sample. Hence, if a mood stabilizer is indicated in patients particularly at risk for hyponatremia, valproate and lithium should be favored in this respect.

Neurohypophyseal release of AVP can be directly stimulated by serotonin (5-HT) and other monoamines. ${ }^{44}$ This mechanism may at least partly underlie the adverse SIADHmediated hyponatremia secondary to antidepressants because most of them, including TCAs, SNRIs, and SSRIs, are potent combined monoamine or selective 5-HT reuptake inhibitors. The TCAs have been among the first psychotropic drug classes in which hyponatremia has been observed. ${ }^{45}$ In our study, hyponatremia prevalence of TCAs was close to the average of all other psychotropic drugs. Of interest, hyponatremia prevalence of SSRI was also not significantly higher than the average. This drug class has been implicated in hyponatremia and falls in many case reports ${ }^{46}$ and a previous case-control study. ${ }^{12}$ Nevertheless, our findings are in line with a previous retrospective study that also failed to detect an increased hyponatremia risk associated with SSRI use. ${ }^{31}$ Accordingly, Letmaier et $\mathrm{al}^{23}$ also found just an average risk for SSRI treatment alone, which significantly increased in case of combination therapy with ACEI, diuretics, and both. Likewise, in our sample, all patients of the fifth quintile (most severe hyponatremia) who received an SSRI were significantly more often comedicated with a thiazide or an ACEI or both than the SSRI cases of the least severe hyponatremic first quintile. Hence, hyponatremia secondary to SSRI treatment may become relevant primarily in the presence of other risk-conveying drugs or conditions. For the SNRI venlafaxine, we found an increased hyponatremia prevalence in accord with prior evidence. ${ }^{2,12,23} \mathrm{~A}$ surprising finding was the strong association of hyponatremia with trazodone treatment. However, the large CI has to be considered. So far, no observations of hyponatremia secondary to trazodone other than in toxic dose ranges ${ }^{4-49}$ have been published.

In a recent review of case reports and case series regarding hyponatremia due to antipsychotic drug treatment, Meulendijks et $\mathrm{al}^{50}$ report on an association of both FGAs and SGAs with hyponatremia. However, systematic studies on this topic are lacking, and polydipsia as a frequent symptom in schizophrenia is a major confounding factor. Our data provide evidence that FGAs are associated with an increased risk of hyponatremia as compared with SGAs. As a matter of fact, it has been suggested that FGA-mediated long-term dopamine $\mathrm{D}_{2}$ receptor blockade may overly sensitize $D_{2}$ receptors, a mechanism known to elevate AVP levels, ${ }^{50}$ which could explain this observation. Second-generation antipsychotics such as clozapine, on the other hand, have low $\mathrm{D}_{2}$ receptor-binding affinities. Thus, $\mathrm{D}_{2}$ 
receptor supersensitivity may be normalized or avoided upon SGA treatment, resulting in less hyponatremia induction. In fact, clozapine is thought to exert even salutary effects on polydipsia-induced hyponatremia in psychotic patients. ${ }^{51-53}$ This would match our observation that, whereas the proportion of treatments with FGAs was equally distributed among the quintiles of increasing hyponatremia severity, SGA treatment was significantly less prevalent in the most severe cases (fifth quintile). However, others did not find such associations between antipsychotic use and hyponatremia. ${ }^{54}$ Thus, more research is warranted regarding this clinically highly relevant topic, and importantly, future studies need to be controlled for polydipsia.

\section{Impact of Other Diagnostic and Treatment-Related Parameters}

We did not find any routine laboratory parameter (except $\left[\mathrm{Cl}^{-}\right]$as the corresponding anion) to be associated with hyponatremia severity in our sample. By contrast, the calculated serum osmolarity showed a high correlation, and the average values of all severity quintiles in the sample were well below the lower physiological limit of $275 \mathrm{mOsm} / \mathrm{kg} \mathrm{H}_{2} \mathrm{O}$ as evidenced by Supplementary Table A (Supplemental Digital Content 1). Although this is not surprising (because the calculation is mainly based on $\left[\mathrm{Na}^{+}\right]$), our data nonetheless provide other indirect evidence of SIADH as an important pathophysiologic factor at least in the fifth quintile comprising the most severe hyponatremia cases: First, the average serum urea and the hematocrit were at the lower end of the reference range. Second, we found a negative correlation between serum osmolarity and eGFR over the complete hyponatremia sample.

We also analyzed treatment-related parameters such as somatic comorbidity and polypharmacy. We were able to show that a higher degree of hyponatremia severity was associated with prescription of higher numbers of nonpsychiatric rather than psychiatric drugs. This association was significant for treatment with ACEIs alone or in combination with thiazides, which is in line with recent data. ${ }^{23}$ We did not find a correlation of hyponatremia severity with the number of prescribed psychiatric drugs or the total number of applied drugs, respectively. However, there was a significant negative correlation of hyponatremia severity and days in hospital treatment, which is probably due to early referral to medical units of symptomatic cases.

In conclusion, age, female sex, and treatment with particular psychiatric drugs (venlafaxine, trazodone, carbamazepine, oxcarbazepine) rather than drug classes as a whole conveyed an elevated hyponatremia risk in our large psychiatric inpatient population. Major determinants for hyponatremia severity were again age and female sex and also polypharmacy with nonpsychiatric drugs, especially ACEI and combined ACEI and thiazide treatment. Besides gastrointestinal symptoms, neuropsychiatric signs such as sedation, confusion, and gait impairment are rather frequent and may occur already in mild cases. Clinicians are advised to measure serum $\left[\mathrm{Na}^{+}\right]$on a regular basis and should be aware of this underrecognized condition in psychiatric inpatients that calls for immediate treatment.

\section{AUTHOR DISCLOSURE INFORMATION}

C.L-A. has received lecture fees and/or travel support from Novartis, AstraZeneca, and Servier and research funding from Pfizer Pharma and Bristol-Myers Squibb. J.C. is on the speakers' board of Servier and has received congress support from Tanita Europe, AstraZeneca, and Janssen-Cilag and research support from Lilly Germany, Pfizer GmbH, Janssen-Cilag,
Servier, and Alpine Biomed. G.K. has received lecture and/or consulting fees from Pfizer, Boehringer, and Mundipharma and research funding from Pfizer, Actavis, and Shire. T.S. has received speaker's honoraria from Pfizer/Eisai, Novartis Pharma, and Merz Pharma. F.H., A.G., and R.G. report no financial disclosures.

\section{REFERENCES}

1. Adrogue HJ, Madias NE. Hyponatremia. N Engl J Med 2000;342:1581-1589.

2. Siegel AJ. Hyponatremia in psychiatric patients: update on evaluation and management. Harv Rev Psychiatry. 2008;16:13-24.

3. Boscoe A, Paramore C, Verbalis JG. Cost of illness of hyponatremia in the United States. Cost Eff Resour Alloc. 2006;4:10.

4. Peri A, Pirozzi N, Parenti G, et al. Hyponatremia and the syndrome of inappropriate secretion of antidiuretic hormone (SIADH). J Endocrinol Invest. 2010;33:671-682.

5. Gross P, Reimann D, Henschkowski J, et al. Treatment of severe hyponatremia: conventional and novel aspects. J Am Soc Nephrol. 2001;12(suppl 17):S10-S14.

6. Reeves WB, Buchet DG, Andreoli TE. Posterior pituitary and water metabolism. In: Wilson J, Foster D, eds. Williams Textbook of Endocrinology. 341-387. 1998. Philadelphia, PA: WB Saunders 1998:341-387.

7. Liamis G, Milionis H, Elisaf M. A review of drug-induced hyponatremia. Am J Kidney Dis. 2008;52:144-153.

8. Kumar S, Berl T. Sodium. Lancet. 1998;352:220-228.

9. Ellison DH, Berl T. Clinical practice. The syndrome of inappropriate antidiuresis. N Engl J Med. 2007;356:2064-2072.

10. Douglas I. Hyponatremia: why it matters, how it presents, how we can manage it. Cleve Clin J Med. 2006;73(suppl 3):S4-S12.

11. Liu BA, Mittmann N, Knowles SR, et al. Hyponatremia and the syndrome of inappropriate secretion of antidiuretic hormone associated with the use of selective serotonin reuptake inhibitors: a review of spontaneous reports. CMAJ. 1996;155:519-527.

12. Movig KL, Leufkens HG, Lenderink AW, et al. Association between antidepressant drug use and hyponatraemia: a case-control study. $\mathrm{Br} J$ Clin Pharmacol. 2002;53:363-369.

13. Roxanas M, Hibbert E, Field M. Venlafaxine hyponatraemia: incidence, mechanism and management. Aust N Z J Psychiatry. 2007;41:411-418.

14. Cosgray RE, Hanna V, Davidhizar RE, et al. The water-intoxicated patient. Arch Psychiatr Nurs. 1990;4:308-312.

15. Miller WR. Psychogenic factors in the polyuria of schizophrenia. JNerv Ment Dis. 1936;84:418-421

16. Asadollahi K, Beeching N, Gill G. Hyponatraemia as a risk factor for hospital mortality. QJM. 2006;99:877-880.

17. de Leon J. Polydipsia - a study in a long-term psychiatric unit. Eur Arch Psychiatry Clin Neurosci. 2003;253:37-39.

18. Kirby D, Harrigan S, Ames D. Hyponatraemia in elderly psychiatric patients treated with selective serotonin reuptake inhibitors and venlafaxine: a retrospective controlled study in an inpatient unit. Int J Geriatr Psychiatry. 2002;17:231-237.

19. Movig KL, Leufkens HG, Lenderink AW, et al. Serotonergic antidepressants associated with an increased risk for hyponatraemia in the elderly. Eur J Clin Pharmacol. 2002;58:143-148.

20. Bhagat CI, Garcia-Webb P, Fletcher E, et al. Calculated vs measured plasma osmolalities revisited. Clin Chem. 1984;30:1703-1705.

21. Lange-Asschenfeldt C, Grohmann R, Lange-Asschenfeldt B, et al. Cutaneous adverse reactions to psychotropic drugs: data from a 
multicenter surveillance program. J Clin Psychiatry. 2009;70: $1258-1265$.

22. Grohmann R, Engel RR, Ruther E, et al. The AMSP drug safety program: methods and global results. Pharmacopsychiatry. 2004;37(suppl 1):S4-S11

23. Letmaier M, Painold A, Holl AK, et al. Hyponatraemia during psychopharmacological treatment: results of a drug surveillance programme. Int J Neuropsychopharmacol. 2012;15:739-748.

24. Upadhyay A, Jaber BL, Madias NE. Incidence and prevalence of hyponatremia. Am J Med. 2006;119:S30-S35.

25. Mannesse CK, Vondeling AM, van Marum RJ, et al. Prevalence of hyponatremia on geriatric wards compared to other settings over four decades: a systematic review. Ageing Res Rev. 2012;12:165-173.

26. Turnheim $\mathrm{K}$. When drug therapy gets old: pharmacokinetics and pharmacodynamics in the elderly. Exp Gerontol. 2003;38:843-853.

27. Anpalahan M. Chronic idiopathic hyponatremia in older people due to syndrome of inappropriate antidiuretic hormone secretion (SIADH) possibly related to aging. J Am Geriatr Soc. 2001;49:788-792.

28. Juul KV, Klein BM, Sandstrom R, et al. Gender difference in antidiuretic response to desmopressin. Am J Physiol Renal Physiol. 2011;300:F1116-F1122.

29. Grikiniene J, Volbekas V, Stakisaitis D. Gender differences of sodium metabolism and hyponatremia as an adverse drug effect. Medicina (Kaunas). 2004;40:935-942.

30. Josiassen RC, Filmyer DM, Geboy AG, et al. Impact of hyponatremia on resource utilization in state psychiatric hospitals: a retrospective analysis. J Clin Psychopharmacol. 2012;32:580-582.

31. Bun S, Serby MJ, Friedmann P. Psychotropic medication use, hyponatremia, and falls in an inpatient population: a retrospective study. J Clin Psychopharmacol. 2011;31:395-397.

32. Renneboog B, Musch W, Vandemergel X, et al. Mild chronic hyponatremia is associated with falls, unsteadiness, and attention deficits. Am J Med. 2006;119:71-78.

33. Tolouian R, Alhamad T, Farazmand M, et al. The correlation of hip fracture and hyponatremia in the elderly. J Nephrol. 2012;25:789-793.

34. Decaux G. Is asymptomatic hyponatremia really asymptomatic? Am J Med. 2006;119:S79-S82.

35. Waikar SS, Mount DB, Curhan GC. Mortality after hospitalization with mild, moderate, and severe hyponatremia. Am J Med. 2009;122:857-865.

36. Sajadieh A, Binici Z, Mouridsen MR, et al. Mild hyponatremia carries a poor prognosis in community subjects. Am J Med. 2009;122:679-686.

37. Verbalis JG, Barsony J, Sugimura Y, et al. Hyponatremia-induced osteoporosis. J Bone Miner Res. 2010;25:554-563.
38. Meinders AE, Cejka V, Robertson GL. The antidiuretic action of carbamazepine in man. Clin Sci Mol Med. 1974;47:289-299.

39. Gold PW, Robertson GL, Ballenger JC, et al. Carbamazepine diminishes the sensitivity of the plasma arginine vasopressin response to osmotic stimulation. J Clin Endocrinol Metab. 1983;57:952-957.

40. Van Amelsvoort T, Bakshi R, Devaux CB, et al. Hyponatremia associated with carbamazepine and oxcarbazepine therapy: a review. Epilepsia. 1994;35:181-188.

41. Kuz GM, Manssourian A. Carbamazepine-induced hyponatremia: assessment of risk factors. Ann Pharmacother. 2005;39:1943-1946.

42. Lin $\mathrm{CH}, \mathrm{Lu} \mathrm{CH}$, Wang FJ, et al. Risk factors of oxcarbazepine-induced hyponatremia in patients with epilepsy. Clin Neuropharmacol. 2010;33:293-296.

43. Dong X, Leppik IE, White J, et al. Hyponatremia from oxcarbazepine and carbamazepine. Neurology. 2005;65:1976-1978.

44. Kis GK, Ocsko T, Galfi M, et al. The effects of orexins on monoaminergic-induced changes in vasopressin level in rat neurohypophyseal cell cultures. Neuropeptides. 2011;45:385-389.

45. Abbott R. Hyponatremia due to antidepressant medications. Ann Emerg Med. 1983;12:708-710.

46. Madhusoodanan S, Bogunovic OJ, Moise D, et al. Hyponatraemia associated with psychotropic medications. A review of the literature and spontaneous reports. Adverse Drug React Toxicol Rev. 2002;21:17-29.

47. Vanpee D, Laloyaux P, Gillet JB. Seizure and hyponatraemia after overdose of trazadone. Am J Emerg Med. 1999;17:430-431.

48. Balestrieri G, Cerudelli B, Ciaccio S, et al. Hyponatraemia and seizure due to overdose of trazodone. $B M J$. 1992;304:686.

49. Baughan E, Jawad SS. Effect of overdose of trazodone. BMJ. 1992;304:1114.

50. Meulendijks D, Mannesse CK, Jansen PA, et al. Antipsychotic-induced hyponatraemia: a systematic review of the published evidence. Drug Saf. 2010;33:101-114.

51. Bersani G, Pesaresi L, Orlandi V, et al. Atypical antipsychotics and polydipsia: a cause or a treatment? Hum Psychopharmacol. 2007;22:103-107.

52. Spears NM, Leadbetter RA, Shutty MS Jr. Clozapine treatment in polydipsia and intermittent hyponatremia. J Clin Psychiatry. 1996;57:123-128.

53. Leadbetter RA, Shutty MS Jr. Differential effects of neuroleptic and clozapine on polydipsia and intermittent hyponatremia. J Clin Psychiatry. 1994;55(suppl b):110-113.

54. Jessani M, Montgomery J, Fedde JD, et al. Lack of association between antipsychotics and hyponatremia in chronic schizophrenia. Schizophr Res. 2006;83:307-309. 